\title{
ANÁLISE FAUNÍSTICA DE MOSCAS-DAS-FRUTAS (DIPTERA, TEPHRITIDAE) EM POMARES DE PESSEGUEIRO EM PORTO ALEGRE, RIO GRANDE DO SUL
}

\author{
Flávio Roberto Mello Garcia ${ }^{1}$ \\ Elio Corseuil ${ }^{2}$
}

\begin{abstract}
FAUNAL STUdy OF FRUIT FLIES (Diptera, TEPHRITIDAE) IN PEACH ORChards IN Porto Alegre, Rio Grande do SUl. In order to characterize the peach trees ecosystem in Porto Alegre related to tephritidea species, a faunal study was developed over the data of the collects of these insects with traps in twelve months. For the characterization of the communities, constancy, abundance and frequency index were determined. Relation between sex were obtained by correlation matrix, sexual proportion and sexual rates. Anastrepha fraterculus (Wiedemann, 1830) was abundant, constant and frequent, Ceratitis capitata (Wiedemann, 1824) was accessory and Anasirepha grandis (Macquart, 1845) accidental. A greater number of females than males was observed.

KEY WORDS. Tephritidae, Anastrepha, Ceratitis capitata, faunal study, peach trees
\end{abstract}

Os Tephritidae, comumente conhecidos como moscas-das-frutas, são tidos como uma das principais pragas da fruticultura da América tropical. O pessegueiro, Prunus persica (L.) (Rosaceae), é uma das espécies vegetais mais suscetíveis ao ataque de moscas-das-frutas (MATIOLI et al. 1989).

O predomínio de Anastrepha fraterculus (Wiedemann, 1830) em diversas frutíferas rosáceas no sul do Brasil foi constatada por: FEHN \& BERTELS (1977); FEHN (1981); LORENZATO $(1984,1988)$ e SALLES \& KOVALESKI (1990).

SILVA et al. (1968) referem seis espécies para o Rio Grande do Sul, das quais apenas A. fraterculus e Ceratitis capitata (Wiedemann, 1824) são referidas como pragas do pessegueiro.

FEHN (1981) em estudo de levantamento da ocorrência das moscas-das-frutas em pessegueiro na área metropolitana de Curitiba e região de Irati, Paraná, coletou 151 exemplares de tefritídeos durante 10 meses de coleta, a maioria fêmeas, exceto para C. capitata onde ocorreu o inverso.

NASCIMENTO \& ZUCCHI (1981) e NASCIMENTO et al. (1983) em estudo de dinâmica populacional de moscas-das-frutas no Recôncavo Baiano em diversas frutíferas constataram que A. fraterculus, Anastrepha obliqua (Macquart, 1845) e Anastrepha sororcula Zucchi, 1978 são as espécies mais comuns.

1) Departamento de Ciências Biológicas e da Saúde, Universidade do Oeste de Santa Catarina. Avenida Senador Attílio Fontana 591-E, Caixa Postal 747, 89809-000 Chapecó, Santa Catarina, Brasil.

E-mail: flaviog@unoesc.rct-sc.br

2) Departamento de Biologia, Pontificia Universidade Católica do Rio Grande do Sul. Avenida Ipiranga 6681, Caixa Postal 1429, 90619 Porto Alegre, Rio Grande do Sul, Brasil. 
Rossi et al. (1988) e MATIOLI et al. $(1988,1989)$ estudando a flutuação populacional de moscas-das-frutas em pessegueiro na região de Caldas, Minas Gerais, concluíram que $C$. capitata foi a espécie dominante.

Em um projeto binacional (Uruguai e Argentina) sobre moscas-das-frutas (FAo 1989), foi constatado que em Salto (Uruguai) a relação total de capturas foi de 1,43 A. fraterculus para 100 C. capitata.

Visando caracterizar o ecossistema de pessegueiro em Porto Alegre com relação as espécies de tefritídeos realizou-se o presente trabalho.

\section{MATERIAL E MÉTODOS}

\section{Caracterização das cultivares}

As coletas foram realizadas em seis pomares das cultivares: Fla 13-72 (com 250 e 600 pés), Premier (200 e 600 pés) e Marli (300 e 350 pés), cujas principais características fenológicas e culturais encontram-se na tabela I, localizados no bairro Vila Nova ( $\left.51^{\circ} 12^{\prime} \mathrm{W} ; 30^{\circ} 07^{\prime} \mathrm{S}\right)$ e Campo Novo $\left(51^{\circ} 11^{\prime} \mathrm{W} ; 30^{\circ} 08^{\prime} \mathrm{S}\right)$, no município de Porto Alegre, Rio Grande do Sul.

A cultivar Fla 13-72 tem seu período de maturação dos frutos da segunda semana de outubro à primeira semana de novembro, produzindo cerca de $17 \mathrm{~kg}$ de fruto por planta. A Premier tem seu período de maturação dos frutos da última semana de outubro até a segunda de novembro produzindo cerca de $25 \mathrm{~kg}$ por planta. A Marli é a cultivar mais tardia pois a maturação ocorre da terceira semana de novembro à terceira semana de dezembro produzindo aproximadamente $30 \mathrm{~kg}$.

Tabela I. Características fenológicas e culturais das três cultivares de pessegueiro utilizadas para a coleta de moscas das-frutas. Porto Alegre, Rio Grande do Sul, 1994/95.

\begin{tabular}{|c|c|c|c|}
\hline Caracteristicas & Fla $13-72$ & Premier & Marli \\
\hline \multicolumn{2}{|c|}{ Pares de flores $/ 25 \mathrm{~cm}$ de ramo $10-12$} & $8-10$ & $12-14$ \\
\hline Forma & $\begin{array}{l}\text { Redonda-ovalada, com } \\
\text { pequena ponta }\end{array}$ & Redonda-ovalada & $\begin{array}{l}\text { Cónica, com sutura } \\
\text { desenvolvida e pequena ponta }\end{array}$ \\
\hline Coloração da polpa & Branco esverdeada & Branco esverdeada & $\begin{array}{l}\text { Esverdeada, com até } 40 \% \text { de } \\
\text { manchas rosadas, e vermelha } \\
\text { ao redor do caroço }\end{array}$ \\
\hline Caroço & Aderente & Semi-livre & Semi-livre \\
\hline Cor da pelicula & $\begin{array}{l}\text { Creme-esverdeada com } 80 \% \\
\text { de vermelho }\end{array}$ & $\begin{array}{l}\text { Creme esverdeada com } 40 \% \\
\text { de vermelho }\end{array}$ & $\begin{array}{l}\text { Esverdeada com até } 40 \% \text { de } \\
\text { vermelho escuro }\end{array}$ \\
\hline $\begin{array}{l}\text { Teor de sólidos solúveis } \\
\left({ }^{\circ} B R I X\right)\end{array}$ & 8 & $9-11$ & $12-14$ \\
\hline Inicio da floraçăo & $30 / \mathrm{VI}$ & $21 N I I$ & $11 / \mathrm{VIII}$ \\
\hline Plena floraçăo & $14 / \mathrm{NII}$ & $11 \mathrm{NIII}$ & $29 / \mathrm{VIII}$ \\
\hline Fim da floração & $28 \mathrm{NII}$ & $18 \mathrm{NIII}$ & $05 / 1 X$ \\
\hline Início da maturaçăo & $14 / x$ & $28 / X$ & $18 / X I$ \\
\hline Fim da maturaçăo & $4 / X I$ & $11 / X \mid$ & $16 / X \|$ \\
\hline Peso dos frutos $(g)$ & $70-80$ & $70-100$ & +100 \\
\hline Produção por planta (Kg) & 1 & 25 & 30,5 \\
\hline Espaçamento (m) & $4 \times 5$ & $4 \times 5$ & $4 \times 5$ \\
\hline Idade (anos) & 6 & 6 & 6 \\
\hline
\end{tabular}

\section{Coleta de adultos em frascos caça-moscas}

Para coleta de adultos foram instaladas armadilhas caça-moscas constituídas de cilíndros plásticos opacos de $21 \mathrm{~cm}$ de comprimento com $8 \mathrm{~cm}$ de diâmetro com quatro orifícios de $1 \mathrm{~cm}$ de diâmetro dispostos simetricamente a uma altura de 15 
$\mathrm{cm}$, distanciados um frasco do outro por aproximadamente $10 \mathrm{~m}$, com $200 \mathrm{ml}$ de solução aquosa de vinagre de vinho tinto a $25 \%$ como atrativo, colocadas a uma altura aproximada de $1 \mathrm{~m} 50 \mathrm{~cm}$, dentro da copa de árvores de pessegueiro.

Os frascos, cinco por pomar, foram utilizados na captura de moscas-das-frutas no período de outubro de 1994 a setembro de 1995. Durante este período foram realizadas visitas semanais aos pomares para troca da solução atrativa e coleta dos tefritídeos, colocando-os em frascos etiquetados, contendo álcool $70 \%$ para posterior separação por sexo, contagem e identificação em laboratório.

\section{Identificação taxonômica}

Ceratitis capitata foi reconhecida por comparação com materiais contidos nas coleções do Museu de Ciências e Tecnologia da Pontifícia Universidade Católica do Rio Grande do Sul (PUCRS), e também utilizando as características diagnósticas apresentadas por ZUCCHI et al. (1993).

O reconhecimento das espécies de Anastrepha Schiner, 1868 foi baseado no ápice do ovipositor das fêmeas, para tanto, algumas fềmeas foram colocadas em posição ventral em uma lâmina sob microscópio estereoscópico, e com auxílio de dois estiletes foi extrovertido o ovipositor. O exame do ovipositor foi feito sob aumento de 40 ou 100 vezes, neste caso, se colocou uma gota de glicerina sobre ovipositor. Posteriormente foi usada a chave dicotômica para espécies de Anastrepha ocorrentes no Brasil.

Foram depositados exemplares de cada espécie coletada no Museu de Ciências e Tecnologia da PUCRS e no Museu Zoobotânico da Universidade do Oeste de Santa Catarina (UNOESC).

\section{Análise faunística das coletas de adultos de moscas-das-frutas}

Foram analisados os índices de constância e abundância em função dos dados de coletas semanais de adultos de moscas-das-frutas, para a caracterização da comunidade, em cada cultivar.

\section{Constância}

A medida faunística de constância foi determinada para cada espécie em cada cultivar estudado, através da fórmula:

$$
C=\frac{p \cdot 100}{N}
$$

onde: (C) constância em porcentagem; (p) número de coletas contendo a espécie; (N) número total de coletas.

As espécies foram agrupadas nas seguintes categorias, levando em conta a constância: espécies constantes, presentes em mais de $50 \%$ das coletas; espécies acessórias, presentes em 25 a $50 \%$ das coletas; espécies acidentais, presentes em menos de $25 \%$ das coletas.

\section{Abundância}

Para calcular a abundância das populações foi empregado uma medida de dispersão, determinando-se o desvio padrão, erro padrão da média e intervalo de confiança da média (I.C.), ao nível de $1 \%$ e $5 \%$ de probabilidade utilizando-se a distribuição em t, definindo-se as seguintes classes de abundância para as espécies 
coletadas: 1) rara: número de indivíduos menor que o limite inferior do I.C. a $1 \%$ probabilidade; 2) dispersa: número de indivíduos situados entre os limites inferiores do I.C. a $5 \%$ e $1 \%$ de probabilidade; 3 ) comum: número de indivíduos situados entre os limites superior e inferior do I.C. a $5 \%$ de probabilidade; 4) abundante: número de indivíduos situados entre os limites superiores do I.C. a $5 \%$ e $1 \%$ de probabilidade; 5) muito abundante: número de indivíduos maior que o limite superior do I.C. a $1 \%$ de probabilidade.

\section{Correlação entre os níveis populacionais de cada sexo}

Para verificar a existência ou não de proporcionalidade entre os níveis populacionais de exemplares de cada sexo para $A$. fraterculus e $C$. capitata fez-se cálculo de matriz de correlação pelo programa Microstat em função dos números semanais de fềmeas e machos.

\section{Proporção sexual}

Calculou-se a proporção sexual para as moscas coletadas em frascos caçamoscas, dividindo-se o número de fêmeas pelo de machos.

Testou-se a hipótese da razão 1:1 entre os sexos através do cálculo do Qui-quadrado.

\section{RESULTADOS E DISCUSSÃO}

\section{Espécies coletadas}

Foi coletado um total de 667 tefritídeos pertencentes a três espécies: $A$. fraterculus - 549 exemplares, C. capitata - 112 exemplares, e A. grandis com apenas seis exemplares cujas datas de coleta com seus respectivos números de exemplares por sexo e cultivar foram: 26/05/95 -1 fêmea e 1 macho (Premier), 02/06/95 -1 fêmea (Fla 13-72); 1 macho (Premier) e 2 fêmeas (Marli).

Os percentuais para cada espécie foram: C. capitata $16,8 \%$, A. grandis $0,9 \%$ e $A$. fraterculus $82,3 \%$.

Dentre as espécies coletadas $A$. grandis é a única que não apresenta o pêssego como hospedeiro, atacando principalmente curcubitáceas (GALLO et. al. 1988), portanto a ocorrência dessa espécie nos pomares de pessegueiro explica-se pela presença de plantações de pepinos e abóboras próximas a alguns pomares.

\section{Abundância}

Através de análise de estatística descritiva chegou-se aos seguintes limites superiores (LS) e limites inferiores (LI) dos intervalo de confiança (IC) para as médias de tefritídeos/mês: IC 5\% LS =2,784; IC 5\% LI=1,139; IC 1\% LS=3,059; IC $1 \% \mathrm{LI}=0,869$.

A partir da comparação das médias de moscas-das-frutas de cada espécie com os intervalos de confiança chegou-se as classificações para cada mês de coleta apresentadas na tabela II.

Pode-se constatar que $A$. fraterculus foi muito abundante no período de frutificação das três cultivares, contudo $C$. capitata somente foi muito abundante na época de maturação da cultivar Marli, sendo esta cultivar provavelmente a mais atacada por essa espécie em Porto Alegre. A espécie $A$. grandis foi rara em todo o período de coleta. 
Tabela II. Classificação das espécies de moscas-das-frutas quanto a abundância nas diversas cultivares de pessegueiro em função da média de moscas/semanas dentro de cada mês. Porto Alegre, Rio Grande do Sul, 1994/95.

\begin{tabular}{|c|c|c|c|c|c|c|}
\hline \multirow[t]{2}{*}{ Mês } & \multicolumn{2}{|c|}{ Fla $13-72$} & \multicolumn{2}{|c|}{ Premier } & \multicolumn{2}{|c|}{ Marli } \\
\hline & A. fraterculus & C. capitata & A. fraterculus & C. capitata & A. fraterculus & C. capitata \\
\hline Outubro & Muito abundante & Rara & Muito abundante & Rara & Comum & Rara \\
\hline Novembro & Muito abundante & Rara & Muito abundante & Rara & Muito abundante & Comum \\
\hline Dezembro & Muito abundante & Dispersa & Muito abundante & Comum & Muito abundante & Muito abundante \\
\hline Janeiro & Rara & Abundante & Abundante & Abundante & Rara & Comum \\
\hline Fevereiro & Rara & Abundante & Rara & Dispersa & Rara & Rara \\
\hline Março & Rara & Dispersa & Rara & Rara & Comum & Rara \\
\hline Abril & Rara & Rara & Comum & Rara & Comum & Rara \\
\hline Maio & Dispersa & Rara & Rara & Rara & Rara & Rara \\
\hline Junho & Rara & Rara & Rara & Rara & Rara & Rara \\
\hline Julho & Rara & Rara & Rara & Rara & Dsipersa & Rara \\
\hline Agosto & Rara & Rara & Rara & Rara & Rara & Rara \\
\hline Setembro & Comum & Rara & Comum & Rara & Comum & Rara \\
\hline
\end{tabular}

\section{Constância}

Através da análise de constância das espécies dentro de cada cultivar chegou-se aos percentuais apresentados na tabela III.

Tabela III. Constância (\%) para as três espécies coletadas nas cultivares de pessegueiro estudadas. Porto Alegre, Rio Grande do Sul, 1994/95.

\begin{tabular}{lccc}
\hline \multicolumn{1}{c}{ Mês } & Fla 13-72 & Premier & Marli \\
\hline Anastrepha grandis & 0,0 & 5,0 & 5,0 \\
Anastrepha fraterculus & 60,0 & 72,5 & 75,0 \\
Ceratitis capitata & 25,0 & 23,8 & 30,0 \\
\hline
\end{tabular}

Constatou-se que $A$. fraterculus foi constante, $C$. capitata foi acessória e $A$. grandis foi acidental nas três cultivares estudadas.

Portanto, A. fraterculus foi a espécie mais abundante, constante e freqüente corroborando os resultados obtidos por FAGUNDES (1967); FEHN \& BERTELS (1977); FEHN (1981); BLEICHER et al. (1982); LORENZATO (1984, 1988), e SALLES \& KOVALESKI (1990), todavia resultados diferentes foram obtidos por MALAVASI et al. (1980); NASCIMENTO \& ZUCCHI (1981); NASCIMENTO et al. (1983); ROSSI et al. (1988); FAO (1989); e MATIOLI et al. $(1988,1989)$ por terem seus estudos sido realizados em outras regiões, com condições fitogeográficas e climáticas diferentes.

\section{Correlação entre os sexos}

Através do cálculo de matriz de correlação constatou-se elevada correlação entre os sexos para $A$. fraterculus, obtendo-se um coeficiente de correlação de 0,93 . Para C. capitata tal relação proporcionou um coeficiente de apenas 0,46 , demostrando assim que, em relação aos sexos há uma uniformidade maior de $A$. fraterculus do que para C. capitata, pois a a flutuação populacional de machos e fêmeas da primeira se assemelharam ao longo do tempo. Outro fator que pode ter influído na análise para $C$. capitata foi o baixo número de insetos coletados e, possivelmente, por uma diferenciada atração entre os sexos pela solução alimentar utilizada como atraente, capturando dessa forma mais fềmeas. 


\section{Proporção sexual}

O número total de exemplares em cada sexo, coletados em frascos caça-moscas, para A. fraterculus foi de 246 machos e 303 fêmeas e para $C$. capitata de 15 machos e 97 fêmeas.

A proporção sexual obtida, nos frascos caça-moscas foi de 1,23 fêmeas para 1 macho de $A$. fraterculus, e de 6,46 fêmeas para 1 macho de $C$. capitata.

Através da análise do Qui-quadrado chegou-se ao valor de 5,918 $(\mathrm{P}<0,05)$ para a proporção sexual em coletas de $A$. fraterculus e de $60,036(\mathrm{P}<0,01)$ para $C$. capitata, contradizendo a proporção esperada de 1:1.

Número superior de fêmeas de $A$. fraterculus já havia sido constatado por FEHN (1981) e SALLES (1995), todavia para C. capitata foi observado por FEHN (1981) número maior de machos, talvez pelo baixa incidência dessa espécie em suas amostragens.

Talvez o atrativo tenha sido mais eficiente na captura de fêmeas como foi observado por ENGELSTEIN \& MORGANTE (1991) para C. capitata utilizando atrativo protéico, o que pode ocorrer inclusive para $A$. fraterculus.

\section{REFERÊNCIAS BIBLIOGRÁFICAS}

Bleicher, J.; D.N. Gassen; L.G. Ribeiro; H. TANAKA, \& A.I. ORTH. 1982. Moscas-das-frutas em macieira e pessegueiro. Florianópolis, EMPASC, 28p.

Engelstein, M. \& J.S. MoRganTE. 1991. Associação entre atrativo alimentar e paraferomonio na captura de "moscas-das-frutas" (Diptera: Tephritidae). Revta bras. Ent. 35 (1): 73-77.

FAGUNDES, A.C. 1967. Coleta e reconhecimento de moscas das frutas. Rev. Fac. Agron. Vet., Porto alegre, (9): 93-109.

FAO. 1989. Avances en las investigaciones sobre moscas de las frutas en el litoral del Rio Uruguay. Uruguai, Ministério de Ganaderia, Agricultura y Pesca de Uruguay, $15 \mathrm{p}$.

FEHN, L M. 1981. Coleta e reconhecimento de moscas das frutas na Região Metropolitana de Curitiba e Irati, Paraná, Brasil. An. Soc. Entomol. Brasil 10 (2): 199-208.

FEHN, L.M. \& A. BERTELS. 1977. Observações sobre armadilhas caça moscas em pomar de pessegueiro em Pelotas - RS. Agros, Pelotas, (12): 31-36.

Gallo, D.; O. NaKano; S. Silveira Neto; R.P.L CaRvalho; G.C.D de Batista; E. BERTI FILHo; J.R.P. PARRA; R.A. ZUCCHI; S.B Alves \& J.D. VENDRAMIN. 1988. Manual de Entomologia Agrícola. São Paulo, Agronômica Ceres, 649p.

LORENZATO, D. 1984 Eficiência de frascos e atrativos no monitoramento e combate de moscas-das-frutas do gênero Anastrepha e Ceratitis capitata. Agron. Sulriograndense, Porto Alegre, 20 (2): 45-62.

1988. Controle integrado de moscas-das-frutas em frutíferas rosáceas. Ipagro Informa, Porto Alegre, (1): 57-70.

MALAVASI, A.; J.S. MORGANTE \& R.A. ZuCCHI. 1980 Biologia de "moscas-dasfrutas"(Diptera: Tephritidae) I: Lista de hospedeiros e ocorrência. Rev. Brasil. Biol. 40 (1): 9-16. 
Matioli, J.C.; M.M. Rossi \& V.H. BuENo. 1988. Suscetibilidade de cultivares de pêssego às moscas das frutas (Diptera: Tephritidae) na região de Caldas - MG. Observações preliminares. An. Soc. Entomol. Brasil. 17 (Supl.): 75-85.

- 1989. Atrativos para Ceratitis capitata (Wied.) em pomar de pessegueiro no município de Caldas - MG. An. Soc. Entomol. Brasil 18 (Supl.): 119-129.

NASCIMENTO, A.S. \& R.A. ZUCCHI. 1981. Dinâmica populacional de moscas-dasfrutas do gênero Anastrepha (Dip.: Tephritidae) no Recôncavo Baiano: I -Levantamento das espécies. Pesq. Agropec. Bras., Brasília, 16 (6): 763-767.

NASCIMENTO, A.S.; R.A. ZUCCHI \& S. SilveIRA NETO. 1983. Dinâmica populacional de moscas-das-frutas do gênero Anastrepha (Dip.: Tephritidae) no Recôncavo Baiano: III - Análise faunística. Pesq. Agropec. Bras., Brasília, 18 (4): 319-328.

Rossi, M.M.; J.C. MATioli \& V.H.P. BueNo. 1988. Principais espécies de moscas-das-frutas (Diptera: Tephritidae) e sua dinâmica populacional em pessegueiro na região de Caldas, sul de Minas Gerais. Rev. Agric., Piracicaba, 63 (3): 329-342.

SALLES, L.A.B. 1995. Estratificação da incidência de Anastrepha fraterculus (Wied.) em fruteiras no sul do Brasil. An. Soc. Entomol. Brasil 24 (3): 423-428.

SAlLES, L.A.B. \& A. KovAlESKI. 1990. Moscas-das-frutas em macieira e pessegueiro no Rio Grande do Sul. Horti Sul, Pelotas, 1 (3): 5-9.

Silva, A. D'A.; C.R. GonçAlves; D.M. Galvão; A.J.L. GonÇAlves; J. Gomes; M. DO N. SiLVA \& L.M. DE SIMONI. 1968. Quarto catálogo dos insetos que vivem nas plantas do Brasil: seus parasitos e predadores. Rio de Janeiro, Ministério da Agricultura, vol. 1, 622p.

ZuCCHI, R.A.; S. Silveira NETO \& O. NAKANO. 1993. Guia de Identificação de Pragas Agrícolas. Piracicaba, Fealq, 139p.

Recebido em 29.VIII.1997; aceito em 04.XII.1998. 